\title{
Optimization of taper structures for III-V on Silicon lasers
}

\author{
D. Van Thourhout ${ }^{1}$, S. Keyvaninia
${ }^{1}$, G. Roelkens
oudene $^{3}$, , G-.H. Duan \\ ${ }^{1}$ Photonics Research Group, INTEC, Ghent University-IMEC, B-9000 Gent, Belgium \\ Phone: +32 92643438 Email: dries.vanthourhout@intec.ugent.be \\ ${ }^{2}$ III-V Lab, a joint lab of 'Alcatel-Lucent Bell Labs France', 'Thales Research and Technology' and 'CEA Leti', Campus Pol- \\ ytechnique, 1, Avenue A. Fresnel, 91767 Palaiseau cedex, France \\ ${ }^{3}$ CEA-LETI, Minatec Campus, 17 Rue des Martyrs, 38054 Grenoble, France
}

\section{Introduction}

Using silicon as a platform for realizing complex integrated photonic circuits is rapidly gaining interest both from the scientific community as from the industry. Tremendous progress in realizing passive devices, high speed modulators and Ge-based detectors has been made over the last decade (for a recent review see [1]). However, efficient light generation directly from silicon, given its indirect bandgap, has not yet been shown. Therefore an alternative approach based on bonding high quality epi-layers on prepatterned silicon waveguide structures has been developed by several groups [2][3][4][5]. We recently proposed a new structure [6] whereby light in the gain section is maximally confined in the III-V quantum well layers. At the ends of the gain section, light is coupled to the silicon waveguide layers using an adiabatic taper. Initial results showed Fabry-Perot type devices operating with threshold currents as low as $30 \mathrm{~mA}$ and output powers up to $4 \mathrm{~mW}$ [6]. Here we present a study focusing on the adiabatic taper and show how its design influences the operation of the device.

\section{Device structure}

Figure 1 shows a top view, longitudinal and transversal cross-sections of the proposed device. Its fabrication and operation was described in [6]. InP-wafers containing the active layers were bonded on prefabricated silicon waveguides with $400 \mathrm{~nm}$ height. Next the InP-substrate was removed and the amplifier structure was defined using deep-UV lithography and plasma etching. Finally the pand n-type contacts were formed. Figure 2 shows a cross-section of a fabricated taper tip.

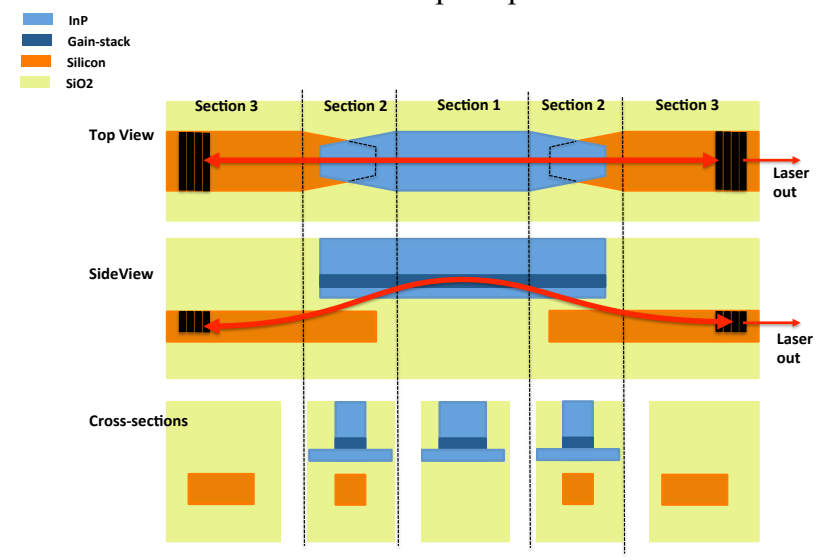

Figure 1 Top view and cross-sections of proposed laser structure.

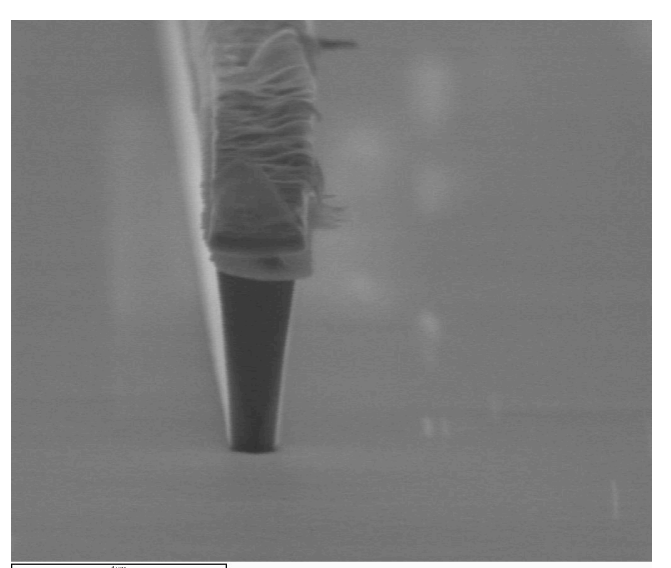

Figure 2 Cross-section of fabricated taper tip

\section{Optimization of taper lengths}

We carried out an extensive design study, investigating the influence of parameters such as taper length, tip width, thickness of the bonding layer and misalignment. Figure 3 shows that for a perfectly fabricated device (green curve) a taper as short as $100 \mu \mathrm{m}$ should allow for over $99 \%$ transmission. However, as soon as there is some misalignment, it is better to increase the length of the taper.

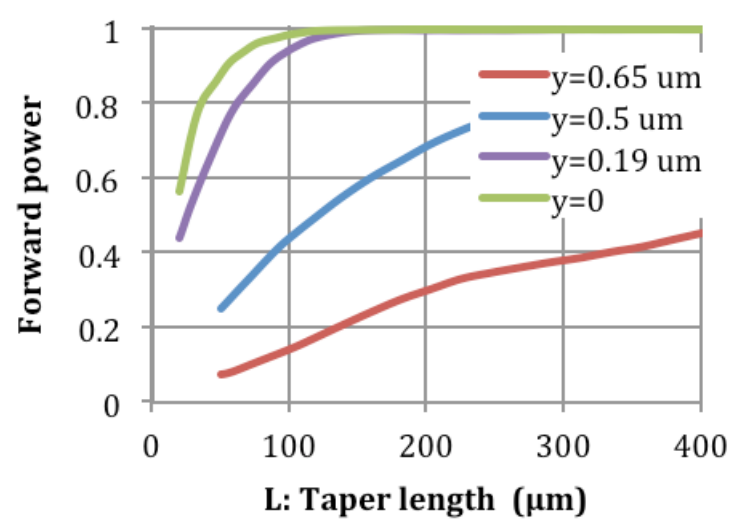

Figure 3 Simulation of power transmitted through tapered transition as function of taper length, and for different values of misalignment $(500 \mathrm{~nm}$ taper tip, $80 \mathrm{~nm}$ bonding layer thickness).

The same holds when calculating the influence of the bonding layer (Figure 4). Again it is clear that increasing the length of the taper leads to a more tolerant structure. 


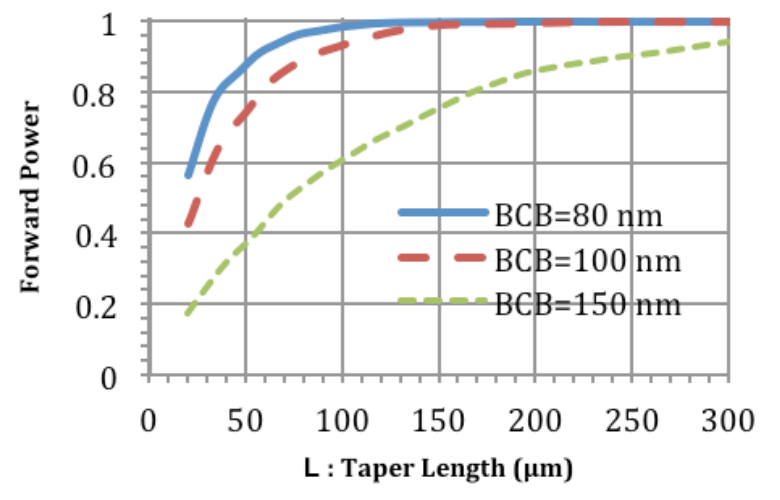

Figure 4 Simulation of power transmitted through tapered transition as function of taper length, and for different values of bonding layer thickness $(500 \mathrm{~nm}$ taper tip). Simulation was carried out for bonding layer with refractive index $1.56(\mathrm{BCB})$, while in the fabricated devices presented in Figure 5 a $\mathrm{SiO}_{2}$ bonding layer was used. This does not influence the overall conclusions however.

Following this design study, we fabricated a series of selected devices with different parameters for active region length and taper length. Figure 5 gives the associated measurement results, for 17 different device designs. In each case the blue curve gives the average value (over 5 devices), the red curve gives the maximum and the green one gives the minimum value.

Overall the shorter devices seem to perform better, not only in terms of threshold current but often also in terms of threshold current density and output power. A length of $150 \mu \mathrm{m}$ seems to be sufficient for the taper, with the device with a $100 \mu \mathrm{m}$ long taper clearly performing worse than all others.

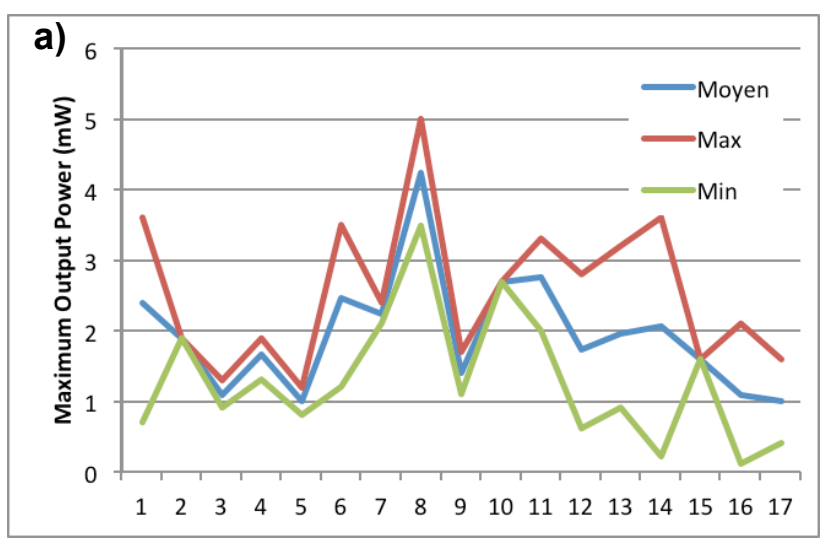

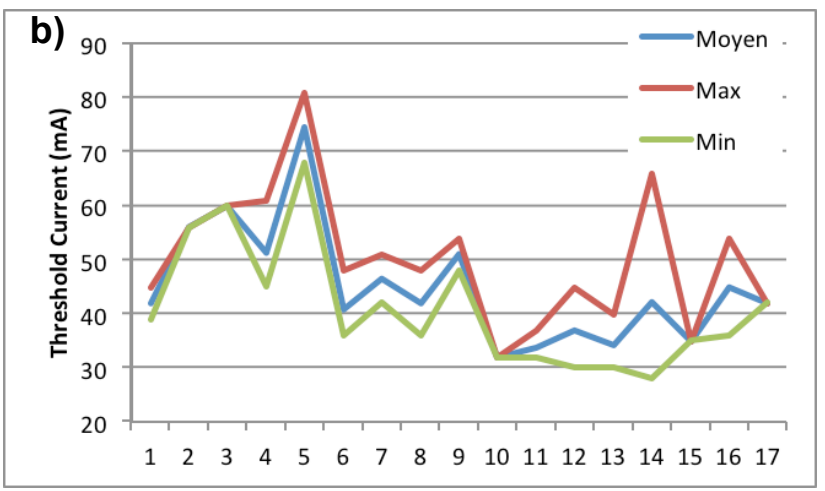

Figure 5 a) Maximum output power and b) threshold current for different laser designs. The horizontal axis denotes the design number. Red/Blue/Green denote maximum/average/minimum values over 5 identical devices per laser design.

\section{Conclusions}

We optimized and experimentally verified the design of an adiabatic taper to be used in a heterogeneous III-V on silicon laser. In addition we showed good reproducibility and yield when comparing identical devices. The optimized gain blocks were combined with a tunable ring resonator and a DBR-mirror to realize a tunable laser. Results will be presented at the conference.

\section{Acknowledgements}

We acknowledge partial financial support by the EU-commission through the FP7 integrated project HELIOS

\section{References}

[1] "Focus Issue Silicon Photonics," Nature Photonics, vol. 4 , no. 8 , pp. 491-578.

[2] A. W. Fang et al., "Single-Wavelength Silicon Evanescent Lasers," IEEE Journal of Selected Topics in Quantum Electronics, vol. 15, no. 3, pp. 535-544, 2009.

[3] G. Roelkens et al., "III-V/silicon photonics for on-chip and intra-chip optical interconnects," Laser \& Photonics Reviews, vol. 4, no. 6, pp. 751-779, 2010.

[4] T. Okumura, T. Maruyama, M. Kanemaru, S. Sakamoto, and S. Arai, "Single-mode operation of GaInAsP/InP-membrane distributed feedback lasers bonded on silicon-on-insulator substrate with rib-waveguide structure," Japanese Journal of Applied Physics Part 2-Letters \& Express Letters, vol. 46, no. 45-49, p. L1206-L1208, Dec. 2007.

[5] J. Van Campenhout et al., "Electrically pumped InP-based microdisk lasers integrated with a nanophotonic silicon-on-insulator waveguide circuit," Optics Express, vol. 15, no. 11, pp. 6744-6749, 2007.

[6] M. Lamponi et al., "Low-Threshold Heterogeneously Integrated InP / SOI Lasers With a Double Adiabatic Taper Coupler," IEEE Photonics Technology Letters, vol. 24 , no. 1, pp. 76-78, 2012. 